\title{
Effects of medial support screws on locking plating of proximal humerus fractures in elderly patients: a retrospective study
}

\author{
Pengcheng Shen ${ }^{1,2}$, Yu Zhu ${ }^{3}$, Lifan $\mathrm{Zhu}^{2}$, Xiaolin $\mathrm{Li}^{4}$, Yaozeng $\mathrm{Xu}^{1}$ \\ ${ }^{1}$ Department of Orthopaedics, The First Affiliated Hospital of Soochow University, Suzhou 215006, China; ${ }^{2}$ Department of Orthopaedics, The Ninth \\ People's Hospital of Suzhou City, Suzhou 215200, China; ${ }^{3}$ Department of Orthopaedics, Suzhou Hospital of Traditional Chinese Medicine, Suzhou \\ 215100, China; ${ }^{4}$ Department of Orthopaedics, Shanghai Sixth People's Hospital Affiliated to Shanghai Jiaotong University, Shanghai 200233, China \\ Contributions: (I) Conception and design: Y Xu; (II) Administrative support: None; (III) Provision of study materials or patients: None; (IV) \\ Collection and assembly of data: L Zhu, X Li; (V) Data analysis and interpretation: P Shen, Y Zhu; (VI) Manuscript writing: All authors; (VII) Final \\ approval of manuscript: All authors. \\ Correspondence to: Dr. Yaozeng Xu. Department of Orthopaedics, The First Affiliated Hospital of Soochow University, 188 Shizi Street, Suzhou \\ 215006, China. Email: xuyaozeng@suda.edu.cn; m13862546422@163.com.
}

\begin{abstract}
Background: This study aimed to explore the effects of medial support screws (MSS) on the locking proximal humeral plate in elderly patients who suffered from proximal humeral fractures.

Methods: From December 2016 to December 2018, eighty-five elderly patients who suffered from proximal humeral fracture and received standard plate or locking plate with or without MSS were selected. The patients were allocated into 3 groups: Standard plate group ( $n=23)$, Locking plate without MSS group ( $n=34)$ and Locking plate with MSS group ( $\mathrm{n}=28$ ). Clinical data from all these 3 groups were collected and analyzed.

Results: These eighty-five elder patients (ranging 60-78 years) accomplished a follow-up with an average of 16.3 months. The outcome data showed that significant difference was found on the Constant score, humeral internal rotation angle and humeral height ratio (all the $\mathrm{P}<0.05$ ) among 3 groups, and a highest Constant score and a lowest humeral internal rotation angle and humeral height ratio loss was revealed in Locking platelet with MSS group. Furthermore, the lowest incidence of post-operation complication events $(7.1 \%, \mathrm{P}=0.051)$ and an evident reduction of secondary surgery incidence $(\mathrm{P}=0.021)$ was also presented in Locking plate with MSS among these 3 groups.
\end{abstract}

Conclusions: The medial support screws in the locking proximal humeral plate in treating proximal humeral fractures could reduce humerus restoration loss and humeral internal rotation angle.

Keywords: Proximal humeral fracture; locking plate; medial support screw; elder patient

Submitted Aug 06, 2019. Accepted for publication Sep 04, 2019.

doi: $10.21037 /$ atm.2019.09.61

View this article at: http://dx.doi.org/10.21037/atm.2019.09.61

\section{Introduction}

Proximal humeral fractures are reported to be the third most universal fracture among elderly patients because of trivial trauma where bones are osteoporotic $(1,2)$. Since the fracture location proximal to shoulder joint, an effective and timely treatment is essential for good prognosis and life quality.

Surgical intervention is often used for unstable fractures, such as displaced 2-part fractures and 3-part and 4-part fractures based on Neer classification, which exhibited a superior prognosis compared to those non-operative methods (3-5). The recent advancement of locking plate technology with the fixed-angle support has been used effectively for proximal humeral fractures so that it has better effects than other fixation methods $(6,7)$. Locking plating is followed by multiple adverse complications, which usually occur in fractures (8-10). Loss of reduction and screw cut-out are the main reasons for revision surgery (11). Thus, mechanical support of the medio-inferior region of the humeral head is essential for the maintenance of fracture 

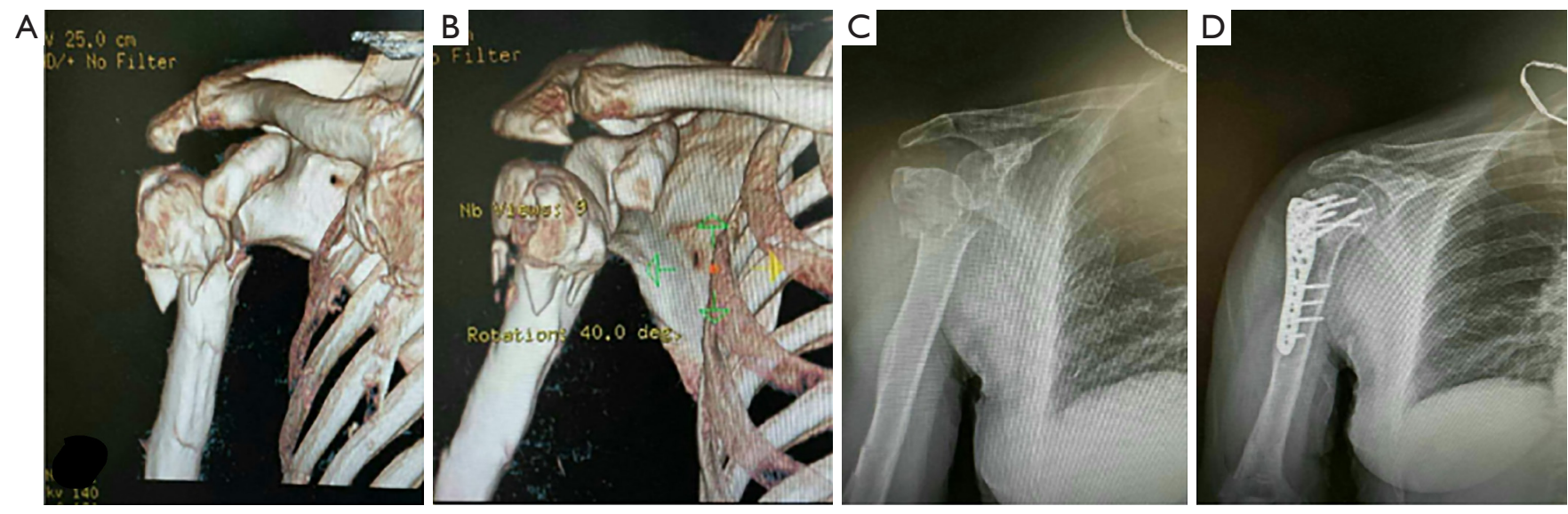

Figure 1 Radiological outcome of locking plate with medial screw support (MSS) in elder patients with proximal humeral fracture. (A,B) Three-dimensional reconstruction by computed tomography; $(\mathrm{C})$ preoperative X-ray image indicated crush fractures on the proximal humerus; (D) postoperative X-ray image of the proximal humeral fracture patients who received locking plate and MSS.

decrease $(8,12)$. However, the therapeutic effects of locking plate with MSS in fracture fixation of older patients remain elucidated.

This study enrolled 85 elderly patients who suffered from proximal humeral fracture and received a standard plate or locking plate alone or with MSS to assess the impacts of MSS in the locking proximal humeral plate.

\section{Methods}

\section{Patients}

We reviewed all the proximal humeral fracture cases in our institution between December 2016 and December 2018. The radiographic and clinical data from all the patients were collected and analyzed. Inclusion criteria were as follows: (I) elderly patients with proximal humeral fracture accompanied with a crush fracture of bone cortex at medial epiphyseal end of humerus shaft were treated with a standard plate or locking plate with or without MSS; (II) three- or four-part fractures or displaced and unstable two-part fractures; (III) age $\geq 60$ years; (IV) follow-up time $\geq 6$ months with complete radiological imaging data at pre- and post-operation. Exclusion criteria: (I) old, pathological, and open fracture; (II) combined with nerve and vascular injury; (III) chronic arthritis. The ethics committee approved this study of our hospital.

\section{Operative techniques}

All the surgeries were performed by the same group of an orthopedic surgeon using brachial plexus block anesthesia or general anesthesia. The patients received image intensifier to obtain intraoperative anteroposterior and axillary views. A deltopectoral approach conducted the open reduction and internal fixation of the fracture with minimal soft tissue dissection. A K-wire under fluoroscopy was employed for reduction. A locking plate was inserted. The distal fragment was fixed by inserting a cortical screw. The proximal segment was stabilized by implanting one or two locking screws. Fluoroscopy was used to check the correct position. Four or 5 locking screws fixed the proximal fragment, and then the medio-inferior region of the humeral head with locking plate and MSS was obliquely plunged by 1 or 2 locking screws (Figure 1). All proximal screws were plunged into the subchondral bone of $5 \mathrm{~mm}$ below. Ethibond sutures (Johnson \& Johnson, Somerville, United States) and the plate was employed to fix the tubercular fragments and rotator cuff tendon. Autograft bone was utilized for the comminuted fractures with a mass deficiency and medial support structure reconstruction. Fluoroscopy was used to measure fracture reduction and screw length. In standard plate group, after extensive exposure of fracture fragments, lag screws and T-type or clover plate were placed to fix, followed by screw length assessment with fluoroscopy, suture closure, and drainage tube placement.

\section{Post-operation treatment}

On the 2 nd-3rd postoperative day, all the patients were required to start with a physiotherapeutic exercise, including 

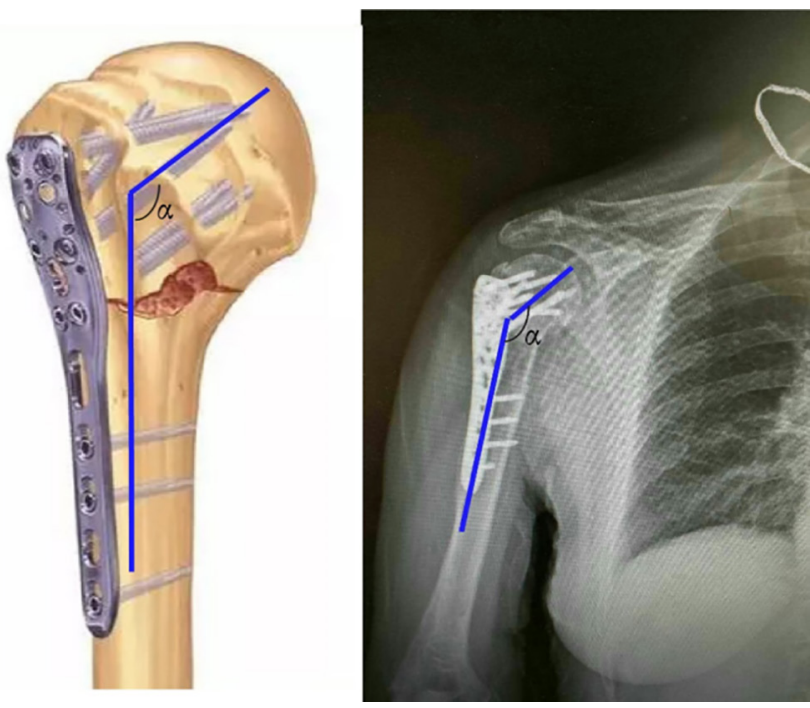

Figure 2 Measurement of humeral internal rotation angle was based on X-ray images and the results was designated as $\Delta \alpha$.

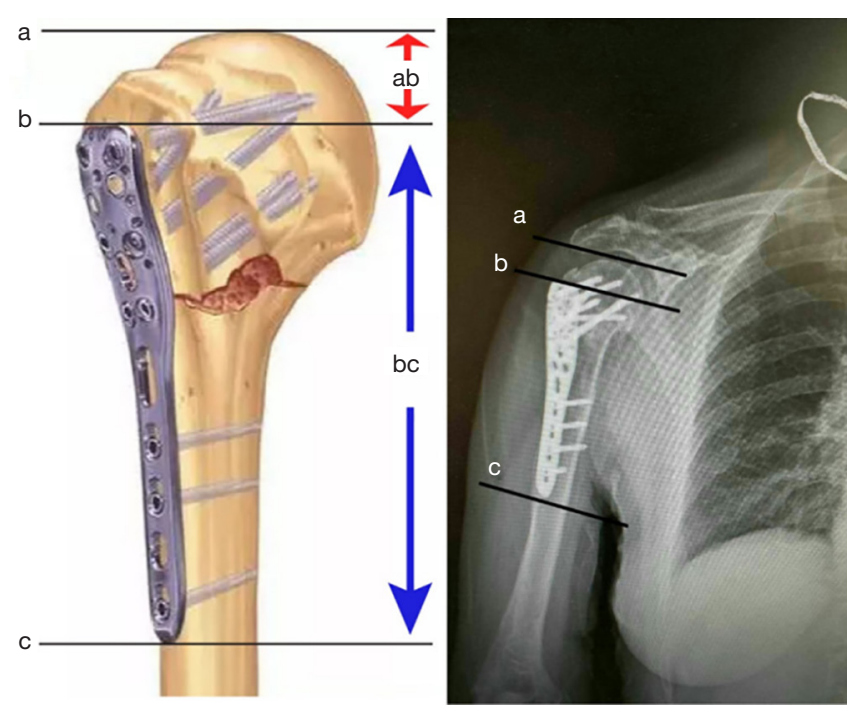

Figure 3 Measurement of humeral height ratio (r) was based on $\mathrm{X}$-ray images. As shown in Figure, three parallel lines perpendicular to longitudinal axis of the medullary cavity was drawn at the level of humerus peak, superior and inferior border of the plate, and was designated as a, b and c, respectively. The distant between line $a$ and $b(a b)$ represented humerus height and the distant between line $\mathrm{b}$ and $\mathrm{c}$ (bc) was plate height. The $\mathrm{r}$ was calculated as humerus height divide plate height. external traction of shoulder joints and arm raising, first passively, followed by movement exercises of the injured arm to a horizontal position at 2 weeks post-operation, and shoulder adduction and internal rotation movement exercises at 3 weeks post-operation. The active movement was then performed after the identification of bridged callus by radiological imaging at $6-8$ weeks post-operation.

\section{Follow-up}

All patients were required for follow-up at 4, 8, and 12 weeks, and 6,12 , and 18 months at post-operation. The follow-up items comprise shoulder function assessment by Constant shoulder score, radiological $\mathrm{X}$-ray imaging, humeral height loss evaluation, internal humeral rotation, the incidence of secondary surgery, and post-operation complications.

For the shoulder function, Constant shoulder score (13), which consisted of 4 subscales: pain (15 points), activities of daily living (20 points), strength (25 points) and range of motion: forward elevation, external rotation, abduction and internal rotation of the shoulder (40 points). The Constant score has a positive correlation with the quality of the function.

The measurement of humeral internal rotation angle was based on X-ray images, and the results were designated as $\Delta \alpha$. As shown in Figure 2, Neck-shaft angle (NSA) of the humerus was assessed at post-operation immediately, and final follow-up and the internal rotation angle was calculated as immediate postoperative NSA minus final NSA.

The measurement of humeral height ratio (r) was conducted based on X-ray images. As shown in Figure 3 three parallel lines perpendicular to the longitudinal axis of the medullary cavity was drawn at the level of humerus peak, superior and inferior border of the plate, and was depicted as $\mathrm{a}, \mathrm{b}$, and $\mathrm{c}$, respectively. The distant between line $\mathrm{a}$ and $\mathrm{b}(\mathrm{ab})$ represented the humeral height, and the distance between line $\mathrm{b}$ and $\mathrm{c}$ (bc) was plate height. The $\mathrm{r}$ was calculated as humeral height divide plate height (Figure 3).

\section{Statistical analysis}

All the statistical analysis was conducted using 18.0 SPSS 
Table 1 Patient demographic data at pre-operation

\begin{tabular}{|c|c|c|c|c|c|}
\hline Characteristics & Standard plate group $(n=23)$ & Locking plate without MSS $(n=34)$ & Locking plate with MSS $(n=28)$ & Statistic & $P$ value \\
\hline Age (yrs) & $61.4 \pm 11.2$ & $62.3 \pm 10.7$ & $64.5 \pm 7.4$ & $F=1.102$ & 0.485 \\
\hline Injury type (n, \%) & & & & $\chi^{2}=3.355$ & 0.521 \\
\hline Traffic accident & $11(12.9)$ & $15(17.6)$ & $13(13.5)$ & & \\
\hline Others & $5(5.8)$ & $12(14.1)$ & $5(5.8)$ & & \\
\hline $\begin{array}{l}\text { Duration between } \\
\text { injury and operation } \\
\text { (days) }\end{array}$ & $4.9 \pm 1.8$ & $5.1 \pm 2.4$ & $5.6 \pm 2.1$ & $\mathrm{~F}=1.514$ & 0.518 \\
\hline Neer classification (n, \% & & & & $\chi^{2}=3.152$ & 0.533 \\
\hline Four-part fracture & $2(2.3)$ & $3(3.5)$ & $5(5.8)$ & & \\
\hline Operative arm (n, \%) & $10(43.5)$ & $15(44.1)$ & $13(46.4)$ & $\chi^{2}=2.761$ & 0.258 \\
\hline
\end{tabular}

MSS, medial support screw.

software (SPSS Inc., Chicago, United States). Comparisons of quantitative data, including age and fracture union, were analyzed using one-way analysis of variance (ANOVA). Constant shoulder score at pre-operation and final followup were compared by using repeated measurement ANOVA. Sum rank test followed with LSD test was utilized to differentiate the humeral internal rotation angle and humeral height ratio. Pearson $\chi^{2}$ test was employed to calculate the data of gender, causes of the fracture, Neer classification, as well as the injury side. A $\mathrm{P}$ value of $\leq 0.05$ was regarded as statistically significant.

\section{Results}

\section{Patient demographic data}

From December 2016 to December 2018, eighty-five elderly patients suffering from proximal humeral fractures received standard plate or locking plate with or without MSS, and the patient demographic data are shown in Table 1. These patients (33 males and 52 females) ranged from $60-78$ years, with the mean age of $65.3 \pm 4.0$ years. The average duration from injury and operation were $6.3 \pm 4.5$, range from 4-13 days. The factors for fracture included $39(45.9 \%)$ cases of traffic accidents, 24 (28.2) falling, 4 (4.7\%) decreasing from a height, $9(10.6 \%)$ exercise injury and $9(10.6 \%)$ others. The fractures were sub-grouped according to Neer classification, including 35 (41.2\%) cases of two-part fractures, $40(47.1 \%)$ cases of three-part fractures and 10 cases $(11.7 \%)$ of four-part fractures [8 (9.4\%) cases of shoulder dislocation].

As shown in Table 1, no significant differences were found on the gender, age, factors for fractures, and Neer classification (all $\mathrm{P}<0.05$ ) among these patients in 3 groups.

\section{Follow-up}

All the eighty-five patients were accomplished with the follow-up ranging from 14-42 months with the mean time of $(16.3 \pm 1.5)$ months. Fracture healing was found in all the patients, and the mean healing time ranged from $10-13$ months with the mean time of $(12.7 \pm 3.7)$ months. Furthermore, no case of nonunion or humerus necrosis was found. No significant difference was found in healing time $(\mathrm{P}=0.561$, Table 2).

\section{Clinical outcome}

The clinical outcome parameters, including Constant score, internal rotation angle $\left(^{\circ}\right)$ and humeral height ratio loss, were assessed, and comparison of these parameters was performed among the different groups. As shown in 
Table 2 Clinical outcome of the patients at pre-operation and post-operation

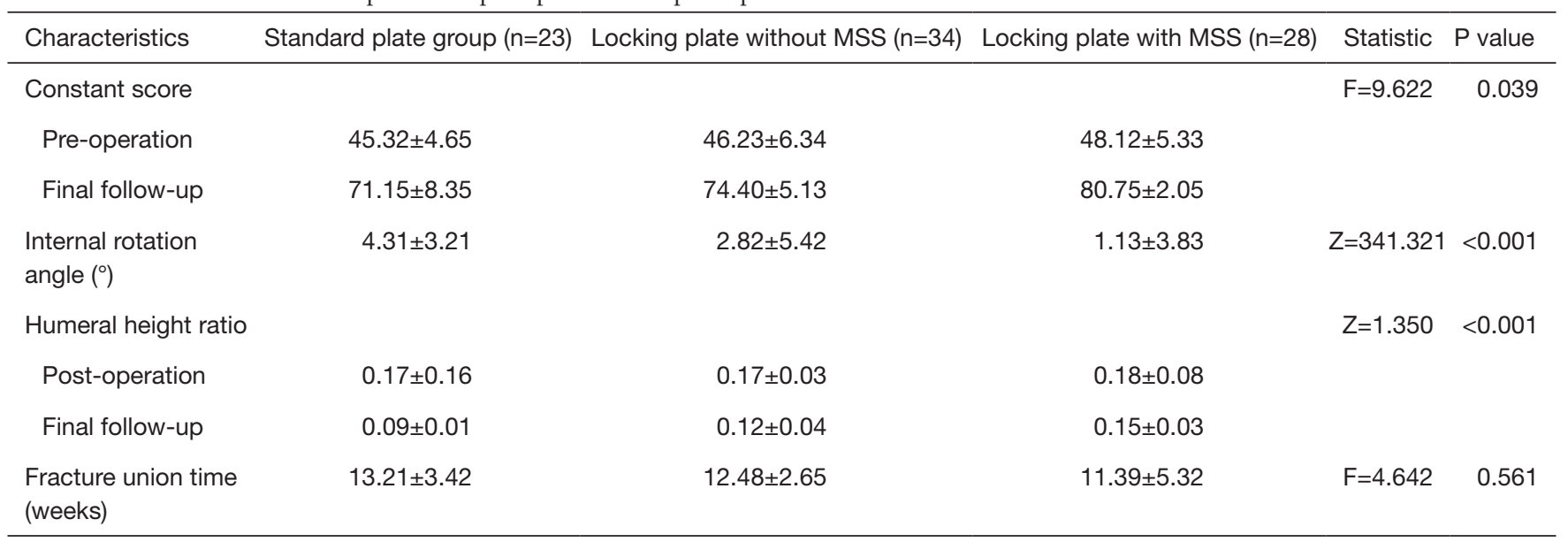

Table 3 Post-operation complications and secondary operation

\begin{tabular}{|c|c|c|c|c|c|}
\hline Characteristics & Standard plate group $(n=23)$ & Locking plate without MSS $(n=34)$ & Locking plate with MSS $(\mathrm{n}=28)$ & Statistic & $P$ value \\
\hline $\begin{array}{l}\text { Acromial } \\
\text { impingement }\end{array}$ & $2(8.6)$ & $4(20.6)$ & $2(7.2)$ & $\chi^{2}=6.451$ & 0.04 \\
\hline Screw perforation & $1(4.3)$ & $3(8.8)$ & $1(3.6)$ & $\chi^{2}=1.373$ & 0.533 \\
\hline $\begin{array}{l}\text { Humeral varus } \\
\text { malunion }\end{array}$ & $4(17.4)$ & $2(5.8)$ & 0 & $\chi^{2}=1.124$ & 0.570 \\
\hline Total & $8(34.7)$ & $10(29.4)$ & $4(14.3)$ & $\chi^{2}=5.941$ & 0.051 \\
\hline Secondary surgery & $7(30.4)$ & $4(10.4)$ & $1(3.6)$ & $\chi^{2}=7.774$ & 0.021 \\
\hline
\end{tabular}

Table 2, the evident difference was shown on the Constant score $(\mathrm{P}<0.05)$, internal rotation angle $\left(^{\circ}\right)(\mathrm{P}<0.001)$ and humeral height ratio loss $(\mathrm{P}<0.001)$ among these 3 groups. Moreover, the results showed that a highest Constant score, and lowest internal rotation angle $\left(^{\circ}\right)$ and humeral height was found in Locking plate with MSS group, secondary of above parameters in Locking plate without MSS group and third in standard plate group $(\mathrm{P}<0.001)$.

\section{Complications}

We also collected the data about post-operation complication and secondary surgery, and the results were shown in Table 3. The incidence of post-operation complications was $4(14.3 \%), 10(29.4 \%)$ and $8(34.7 \%)$, respectively in 3 groups. The manifestations were acromial impingement, screw perforation, humeral head necrosis, and humeral varus malunion. Also, the presence of secondary surgery in Locking plate with MSS group, locking plate without MSS group and standard plate group was 1 (3.6\%), $4(10.4 \%)$ and 7 (30.4\%), respectively.

\section{Discussion}

In the present study, we demonstrated that locking plate with MSS could exert significant benefits on the Constant score, humeral internal rotation angle and humeral height ratio compared to those patients without MSS or with standard plate treatment. Furthermore, the lowest incidence of post-operation complication events and a significantly decreased secondary surgery incidence were also shown in patients treated with locking plate with MSS in comparison 
to those patients without MSS or with standard plate treatment.

In current clinical practice, the stable reduction is of critical significance in fracture healing and for early functional recovery of the shoulder. However, challenges could be presented when application operative stabilization in patients suffering from osteoporotic bone and comminuted fractures, thereby resulting in the controversies about effective therapy for displaced and unstable fractures. Blade-plate fixation, compared to locking plate fixation, displayed advantages associated with the locking plate in treating the proximal humeral fractures (14). The advantages of locking plate were as follow: (I) These plates are biomechanical with low profile better suited for fixing proximal humerus fracture (15). (II) These plates display angular stability and locking screw anchorage (16). (III) These plates provide small holes to fasten rotator cuff besides multiple locking screws. (IV) These plates offer several locking screw options for increased pull-out strength $(11,15)$.

The MSS inserted into medio-inferior region of the humeral head is helpful for the medial column (17-19). Zhang et al. revealed that medial cortical support for anatomic reduction remains the toughest construct (20). Moreover, MSSs in the locking plate and reconstructing the medial column support in proximal humerus fractures could promote humeral head stability and decrease implant failure. Furthermore, the number of support screws could also impact the therapeutic results. Erhardt et al. (21) suggested that at least 5 screws inserted into the head fragment in a locking plate in proximal humerus fractures with disrupted medial hinge could achieve better therapeutic efficacy. Also, Bae et al. (22) indicated that strut graft augmentation elevates the maximum failure load and the initial stiffness of this construct in comparison to a locking plate alone. Here, our results support the beneficial effects exerted by MSS in locking plate for humerus fractures, which is consistent with previous studies.

Despite the advantages of locking plate, complications, including intra-articular screw perforation, subacromial impingement, and fracture re-displacement, have been reported. Thanasas et al. (11) found an avascular necrosis rate of $7.9 \%$, a screw cutout rate of $11.6 \%$, and a reoperation rate of $13.7 \%$, indicating that procedure-related complication rate is high. Additionally, the significance of medial column support for the proximal humerus to decrease the rate of possible complications has been identified $(9,11,16,23)$. Gardner et al (9) identified that a deficient medial support results in $30 \%$ screw perforations in comparison to $6 \%$ for fractures with an intact medial support, which suggests the effectiveness of the inferomedial screw in support of the medial column and maintenance of fracture reduction, especially in medial comminution due to prevention of other implant-related complications from development. Hepp et al. (18) reported that surgical stabilization techniques with the inferomedial trabecular network could be utilized for screw fixation because of the mechanical quality of this region. This inferomedial trabecular network is exploited for locking screw fixation because of its high mechanical strength, which is helpful for osteoporotic bone and prevention of related complications. In the present study, we found a $7.1 \%$ post-operation complication rate and $3.5 \%$ secondary surgery rate.

Although the benefits of MSS in the locking plate in treating proximal humeral fracture have been verified, there are also several limitations in the present study. Firstly, current intraoperative MSS placement was mainly based on the surgical experience and clinical characteristics of the patients. The further systematical analysis of the MSS position in the humerus might be helpful in prognosis. Secondly, the pain improvement data was not collected here during the follow-up, which might underscore the benefits of current surgical methods. Therefore, a large-scale study is required soon to analyze these factors further.

Collectively, MSS in the locking proximal humeral plate in treating elderly patients who suffered from proximal humeral fractures reduce humeral restoration loss and humeral internal rotation angle, which could help treat proximal humeral fractures.

\section{Acknowledgments}

Funding: The present study was funded by National Nature Science Foundation of China to Yaozeng Xu (81472077 and 81672238).

\section{Footnote}

Conflicts of Interest: The authors have no conflicts of interest to declare.

Ethical Statement: The study was conducted after receiving written informed consent from the patient. This research was approved by the Institutional Ethics Committee of the first affiliated hospital of Soochow University. The authors are accountable for all aspects of the work in ensuring that questions related to the accuracy or integrity of any part of 
the work are appropriately investigated and resolved.

\section{References}

1. Court-Brown CM, Caesar B. Epidemiology of adult fractures: A review. Injury 2006;37:691-7.

2. Krappinger D, Bizzotto N, Riedmann S, et al. Predicting failure after surgical fixation of proximal humerus fractures. Injury 2011;42:1283-8.

3. Nho SJ, Brophy RH, Barker JU, et al. Management of proximal humeral fractures based on current literature. J Bone Joint Surg Am 2007;89 Suppl 3:44-58.

4. Park MC, Murthi AM, Roth NS, et al. Two-part and three-part fractures of the proximal humerus treated with suture fixation. J Orthop Trauma 2003;17:319-25.

5. Mighell MA, Kolm GP, Collinge CA, et al. Outcomes of hemiarthroplasty for fractures of the proximal humerus. J Shoulder Elbow Surg 2003;12:569-77.

6. Gardner MJ, Griffith MH, Demetrakopoulos D, et al. Hybrid locked plating of osteoporotic fractures of the humerus. J Bone Joint Surg Am 2006;88:1962-7.

7. Roderer G, Gebhard F, Krischak G, et al. Biomechanical in vitro assessment of fixed angle plating using a new concept of locking for the treatment of osteoporotic proximal humerus fractures. Int Orthop 2011;35:535-41.

8. Agudelo J, Schurmann M, Stahel P, et al. Analysis of efficacy and failure in proximal humerus fractures treated with locking plates. J Orthop Trauma 2007;21:676-81.

9. Gardner MJ, Weil Y, Barker JU, et al. The importance of medial support in locked plating of proximal humerus fractures. J Orthop Trauma 2007;21:185-91.

10. Volgas DA, Stannard JP, Alonso JE. Nonunions of the humerus. Clin Orthop Relat Res 2004;(419):46-50.

11. Thanasas C, Kontakis G, Angoules A, et al. Treatment of proximal humerus fractures with locking plates: a systematic review. J Shoulder Elbow Surg 2009;18:837-44.

12. Lescheid J, Zdero R, Shah S, et al. The biomechanics of locked plating for repairing proximal humerus fractures with or without medial cortical support. J Trauma 2010;69:1235-42.

Cite this article as: Shen P, Zhu Y, Zhu L, Li X, Xu Y. Effects of medial support screws on locking plating of proximal humerus fractures in elderly patients: a retrospective study. Ann Transl Med 2019;7(20):560. doi: 10.21037/atm.2019.09.61
13. Constant CR, Murley AH. A clinical method of functional assessment of the shoulder. Clin Orthop Relat Res 1987;(214):160-4.

14. Siffri PC, Peindl RD, Coley ER, et al. Biomechanical analysis of blade plate versus locking plate fixation for a proximal humerus fracture: comparison using cadaveric and synthetic humeri. J Orthop Trauma 2006;20:547-54.

15. Strohm PC, Helwig P, Konrad G, et al. Locking plates in proximal humerus fractures. Acta Chir Orthop Traumatol Cech 2007;74:410-5.

16. Moonot P, Ashwood N, Hamlet M. Early results for treatment of three- and four-part fractures of the proximal humerus using the PHILOS plate system. J Bone Joint Surg Br 2007;89:1206-9.

17. Clavert P, Zerah M, Krier J, et al. Finite element analysis of the strain distribution in the humeral head tubercles during abduction: comparison of young and osteoporotic bone. Surg Radiol Anat 2006;28:581-7.

18. Hepp P, Lill H, Bail H, et al. Where should implants be anchored in the humeral head? Clin Orthop Relat Res 2003:139-47.

19. Liew AS, Johnson JA, Patterson SD, et al. Effect of screw placement on fixation in the humeral head. J Shoulder Elbow Surg 2000;9:423-6.

20. Zhang $\mathrm{W}$, Zeng L, Liu $\mathrm{Y}$, et al. The mechanical benefit of medial support screws in locking plating of proximal humerus fractures. PLoS One 2014;9:e103297.

21. Erhardt JB, Stoffel K, Kampshoff J, et al. The position and number of screws influence screw perforation of the humeral head in modern locking plates: a cadaver study. J Orthop Trauma 2012;26:e188-92.

22. Bae JH, Oh JK, Chon CS, et al. The biomechanical performance of locking plate fixation with intramedullary fibular strut graft augmentation in the treatment of unstable fractures of the proximal humerus. J Bone Joint Surg Br 2011;93:937-41.

23. Gerber C, Werner CM, Vienne P. Internal fixation of complex fractures of the proximal humerus. J Bone Joint Surg Br 2004;86:848-55. 